\title{
HIGH RESOLUTION AIRBORNE LASER SCANNING AND HYPERSPECTRAL IMAGING WITH A SMALL UAV PLATFORM
}

\author{
Michal Gallay a *, Christoph Eck ${ }^{\mathrm{b}}$, Carlo Zgraggen ${ }^{\mathrm{b}}$, Ján Kaňuk ${ }^{\mathrm{a}}$, Eduard Dvorný ${ }^{\mathrm{a}}$, \\ a Institute of Geography, Faculty of Science, Pavol Jozef Šafárik University in Košice, Jesenná 5, 04001 Košice, Slovakia - \\ \{michal.gallay, jan.kanuk, eduard.dvorny\}@upjs.sk \\ ${ }^{\mathrm{b}}$ Aeroscout GmbH, Technikumstrasse 21, 6048 Horw, Switzerland - \{eck,zgraggen $\} @$ aeroscout.ch
}

Commission I, WG I/Vb

KEY WORDS: payload integration, unmanned aerial system, UAV/VTOL, LiDAR, 3D mapping, terrain model, point density

\begin{abstract}
:
The capabilities of unmanned airborne systems (UAS) have become diverse with the recent development of lightweight remote sensing instruments. In this paper, we demonstrate our custom integration of the state-of-the-art technologies within an unmanned aerial platform capable of high-resolution and high-accuracy laser scanning, hyperspectral imaging, and photographic imaging. The technological solution comprises the latest development of a completely autonomous, unmanned helicopter by Aeroscout, the Scout B1-100 UAV helicopter. The helicopter is powered by a gasoline two-stroke engine and it allows for integrating $18 \mathrm{~kg}$ of a customized payload unit. The whole system is modular providing flexibility of payload options, which comprises the main advantage of the UAS. The UAS integrates two kinds of payloads which can be altered. Both payloads integrate a GPS/IMU with a dual GPS antenna configuration provided by OXTS for accurate navigation and position measurements during the data acquisition. The first payload comprises a VUX-1 laser scanner by RIEGL and a Sony A6000 E-Mount photo camera. The second payload for hyperspectral scanning integrates a push-broom imager AISA KESTREL 10 by SPECIM. The UAS was designed for research of various aspects of landscape dynamics (landslides, erosion, flooding, or phenology) in high spectral and spatial resolution.
\end{abstract}

\section{INTRODUCTION}

The capabilities of unmanned airborne systems (UAS) have become diverse with the recent development of lightweight remote sensing instruments (Colomina and Molina, 2014). In this paper, we demonstrate our custom integration of the state-of-theart technologies within an unmanned aerial platform capable of high-resolution and high-accuracy laser scanning, hyperspectral imaging, and photographic imaging. Reducing the size and weight of laser scanner allows for their integration on UAV platforms (Wallace et al., 2014; Yang and Chen, 2015; Mandlburger et al., 2016). Hyperspectral remote sensing, also known as imaging spectroscopy, is a relatively new technology for UAV platforms that has been used in detection of vegetation species and biomass (Aasen, H. et al., 2015) or precision agriculture (Zarco-Tejada et al., 2013; Bareth et al., 2015; Sima et al., 2016). Other applications of an UAV based hyperspectral imaging can be inferred from manned systems (Ryan et al., 2014; Black et al., 2016; Shendryk et al., 2016).

The presented UAS is built on an industrial basis with an empty weight of the helicopter mechanics of about $47 \mathrm{~kg}$ and a maximum take-off weight MTOW of $77 \mathrm{~kg}$. The flight performance of the helicopter is superior to experienced helicopter pilots and landing within submetre accuracy is possible. Due to the classical helicopter configuration, the scanning performance and homogeneity of data collection in continuous forward flight are superior to multirotor aircraft, in particular under changing wind conditions (wind gusts, side wind, etc.). Production of the presented UAS and payload integration was financed within the project of University Science Park TECHNICOM co-funded by the European Union Structural Funds and the Ministry of
Education, Science, Research and Sport of the Slovak Republic, the executive authority for the Operational Programme Research and Development.

\section{TECHNOLOGICAL SOLUTION}

\subsection{UAV platform}

The technological solution comprises the latest development of a completely autonomous, unmanned helicopter by Aeroscout, the Scout B1-100 UAV helicopter. This helicopter has a $3.2 \mathrm{~m}$ rotor diameter powered by an air-cooled $100 \mathrm{ccm}$ gasoline engine with electric starter and provides a payload capacity of up to $30 \mathrm{~kg}$ (Fig. 1).

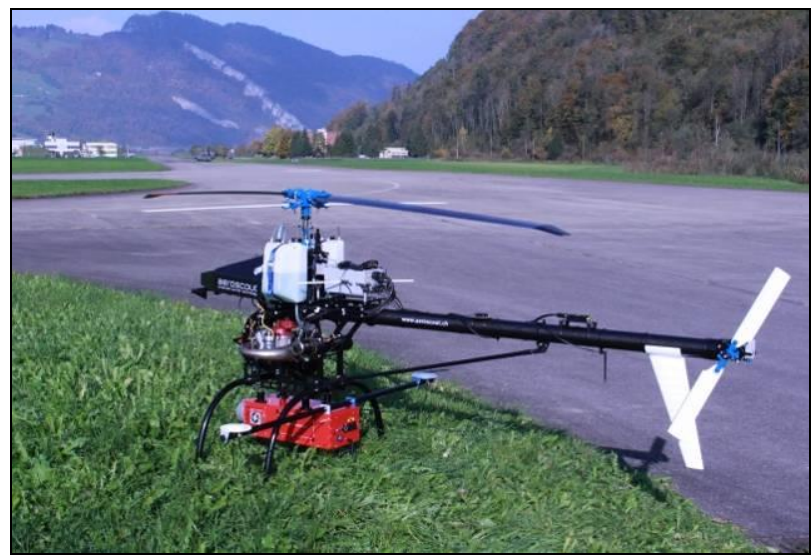

Figure 1: The Scout B1-100 UAV with the integrated laser scanning payload.

\footnotetext{
* Corresponding author
} 
This payload capacity (at about 500m AMSL) includes 101 of fuel $(8 \mathrm{~kg})$, the flight control system $(2 \mathrm{~kg})$, redundant battery power supply $(1 \mathrm{~kg})$, the data link $(1 \mathrm{~kg})$ and a customized payload section $(18 \mathrm{~kg})$. The UAV helicopter is water-resistant and can be operated from $-7{ }^{\circ} \mathrm{C}$ to $+40{ }^{\circ} \mathrm{C}$ even under light rain or windy conditions. The on-board flight control system allows automatic "homing" as well as landing and shut-down of the engine in case of data link failure. The flight endurance can achieve up to 90 minutes, depending on the mission profile. The whole system (Fig. 1) is easily taken apart and put together providing flexibility of payload options, which comprises the main advantage of the UAS. The Scout B1-100 UAV platform was used in several studies (Eck and Imbach, 2011; Yang and Chen, 2015)

\subsection{Laser scanning payload}

The first payload is dedicated for LiDAR data acquisition coupled with visible high resolution imagery. It comprises a VUX-1 laser scanner by RIEGL and a Sony A6000 E-Mount photo camera (Fig. 2). The laser scanner is capable of up to 550,000 measurements per second and demonstrates the cutting edge LiDAR technology of online waveform processing and multiple-time-around processing which provides echo signal digitization of practically unlimited number of targets echoes.

The Airborne Laser Scanning and Monitoring Integration (ALMI) technology has been developed by Aeroscout GmbH in Switzerland for professional 3-D aerial laser scanning based on an UAV system. It allows continuously monitored, accuracy controlled, vibration-isolated, and time-synchronized 3-D laser scanning from the UAV system. The ALMI technology has been developed, tested, and successfully demonstrated on the Aeroscout Scout B1-100 UAV helicopter with different RIEGL laser scanners already, such as the VQ-480-U or the former version LMS Q160. In addition, the ALMI technology can also be applied on other UAV systems or even manned aircraft.

The payload section includes the laser scanner tightly coupled with a high-grade dual-GPS antenna INS/GPS navigation system as well as a GPS reference station. Aeroscout started

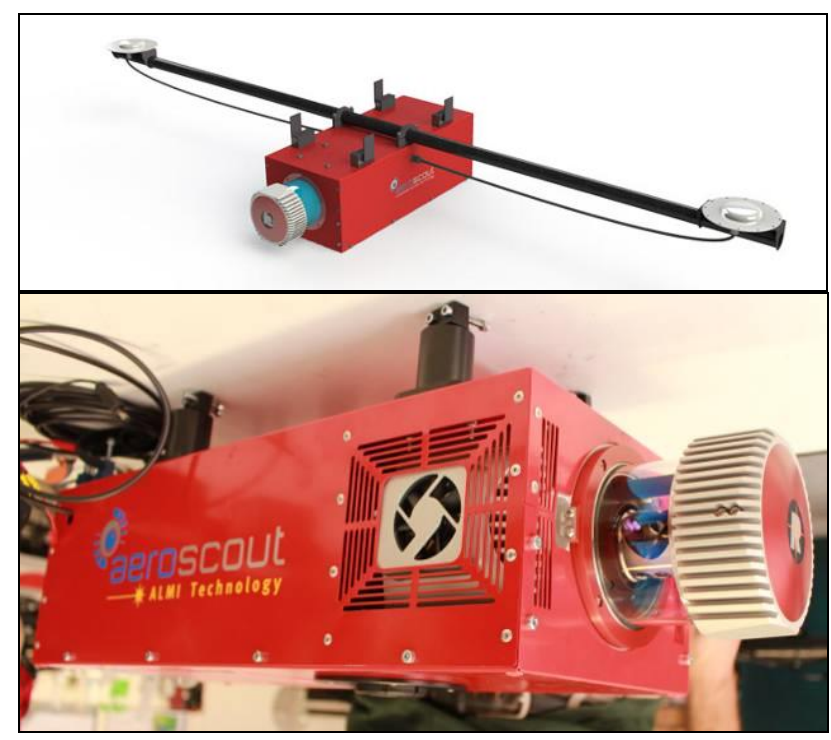

Figure 2: Airborne Laser Scanning and Monitoring Integration (ALMI) of a RIEGL VUX-1 laser scanner, full-frame photo camera, and two GPS antennas. developing the ALMI technology as a contributor to the EU research project "BACS" at ETH Zürich (2006-2010). In the last years, the knowledge was transferred into hardware and software for the industrial requirements of airborne laser scanning. One of the significant advantages of the ALMI technology is to give online feedback already during the flight of the UAV helicopter in respect of data quality, data recording, and time synchronization, as well as network communication conditions (Fig. 3). Depending on the UAV mission profile (altitude above ground, forward speed, etc.) and the laser settings (measurement rate, accuracy requirements, etc.), the laser point density of a single scan line can easily be adjusted from 4 to $400 \mathrm{pts} / \mathrm{m}^{2}$ or more.

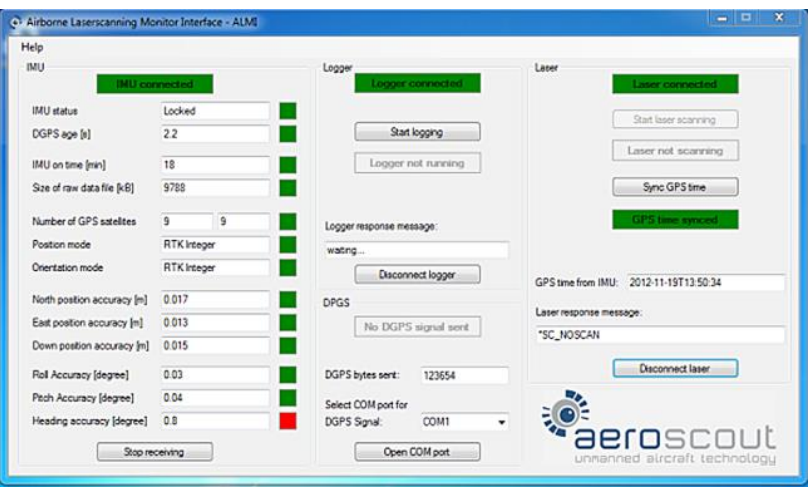

Figure 3: User interface of the Aeroscout ALMI Technology developed for 3D laser recording with DGPS/IMU network configuration.

\subsection{Hyperspectral imaging payload}

The second payload is dedicated for hyperspectral scanning in the spectral range of $400-1000 \mathrm{~nm}$. It comprises an AISA KESTREL 10 camera by SPECIM which is a push-broom imager with high light throughput with an outstanding spatial resolution of 2048 pixels per line. The payload provides radiometrically and spectrally stable data and high signal-tonoise ratio in variable real world remote sensing conditions. The KESTREL camera is a new generation of low-weight hyperspectral cameras (Tab. 1) intended for UAV's and other platforms of limited payload size (weight and volume) (Fig. 4).

\begin{tabular}{|c|c|}
\hline Spectral range & $400-1000 \mathrm{~nm}$ \\
\hline Focal length & F/2.4 \\
\hline Spectral sampling & $1.75 / 3.5 / 7 \mathrm{~nm}$ \\
\hline Frame rate & up to $130 \mathrm{~Hz}$ \\
\hline Signal-to-noise ratio (peak) & $400-800$ \\
\hline Spatial resolution & 1312 or 2048 pixels \\
\hline FOV & $40^{\circ}$ \\
\hline $\begin{array}{c}\text { Total System Power (camera, } \\
\text { GNSS/IMU, DPU) }\end{array}$ & $<41 \mathrm{~W}$ \\
\hline
\end{tabular}

Table 1: Parameters of the hyperspectral camera.

\subsection{Payload integration}

The UAS integrates two kinds of payloads which can be altered. Both payloads integrate a GPS/IMU xNAV550 with dual GPS antenna configuration provided by OXTS for accurate navigation data, i.e. position and attitude measurements of the 
sensor during the data acquisition flight. The payload integration also includes the vibration decoupling of sensitive electronics from the on-board sources of vibration such as fuel engine, main rotor, or tail rotor rpm. Furthermore, the ground control station is equipped with the GPS reference station, allowing the real-time transmission of DGPS correction data. The integration of the payloads and other electronic devices within the UAS allows for on-board data storage as well as broadband data transmission to the ground control station and data visualization during the flight. The UAS was designed for monitoring various aspects of landscape dynamics in high spectral and spatial resolution, for example, landslides, soil erosion, flooding, or vegetation condition.

\section{MISSION PLANNING AND DATA ACQUISITION}

The UAV mission parameters allow forward speed settings from typically 0 up to $15 \mathrm{~m} / \mathrm{s}$ and flight altitude above ground is limited by the required sensor resolution and the sensor specification (e.g., maximal measurement range of the laser scanner, depending on measurement rate). A high resolution laser point cloud typically results from $50 \mathrm{~m}$ flight altitude above ground, a forward speed of $5 \mathrm{~m} / \mathrm{s}$ which yields a point to point density of about $6 \mathrm{~cm}$. The performance of the UAS was tested in several missions near Lucerne, Switzerland. We present the results of testing the LiDAR payload. The parameters are summarized in Table 2 .

\begin{tabular}{|c|c|}
\hline Flying height & $30 \mathrm{~m}$ \\
\hline Area & $2 \mathrm{ha}$ \\
\hline Scanning rate & $550 \mathrm{kHz}$ \\
\hline Point density & $1111 \mathrm{points} / \mathrm{m}^{2}$ \\
\hline Point spacing & $0.03 \mathrm{~m}$ \\
\hline Exposure station baseline & $8 \mathrm{~m}$ \\
\hline
\end{tabular}

Table 2: Summary of the laser scanning mission.

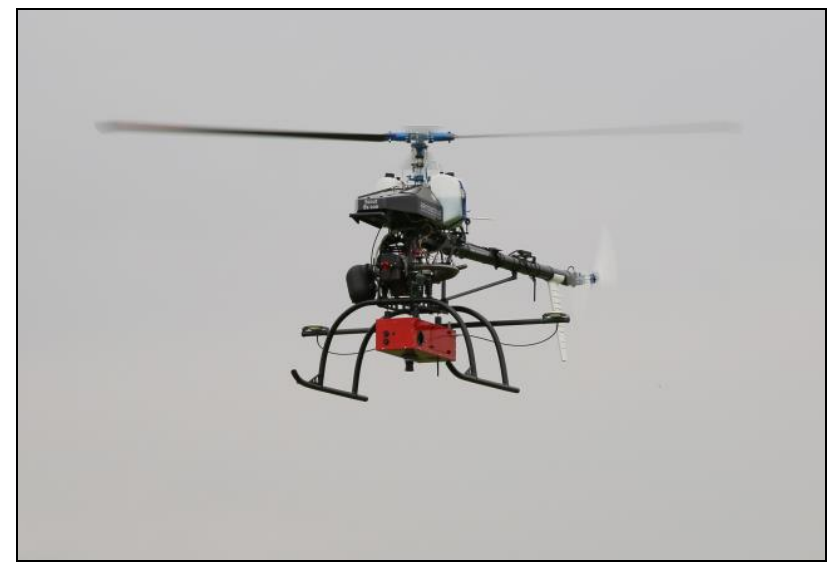

Figure 4: The Scout B1-100 UAV helicopter equipped with the hyperspectral camera during data recording.

\begin{tabular}{|c|c|}
\hline Flying height & $140 \mathrm{~m}$ \\
\hline Area & $2 \mathrm{ha}$ \\
\hline Range of terrain elevation & $3 \mathrm{~m}$ \\
\hline Number of spectral bins & 92 \\
\hline Integration time & $19 \mathrm{~ms}$ \\
\hline Frame rate & $50 \mathrm{~Hz}$ \\
\hline Width of spectral bands & $6.9 \mathrm{~nm}$ \\
\hline Spatial resolution & $0.1 \mathrm{~m}$ \\
\hline
\end{tabular}

Table 3: Summary of the hyperspectral scanning mission.
The UAV flights with the hyperspectral camera were performed in October 2015 in a rural area close to Lucerne, Switzerland. The performance of the hyperspectral scanning payload solution were based on the mission parameters given in Table 3 .

\section{DATA PROCESSING}

\subsection{Laser data processing}

The processing of the laser data was done with the RIEGL software package RiPROCESS. The raw laser data is combined with the position and attitude data collected from the time synchronized onboard IMU/GPS navigation sensor to get the 3D point cloud. Multiple flight lines with overlapping data allow for adjusting the laser data strips to improve the accuracy of their relative registration. The pictures from the photo camera are processed to assign the LiDAR data points with natural colours as RGB values.

\subsection{Hyperspectral data processing}

The hyperspectral raw data were converted to radiance data and geocorrected data using the CaliGeoPRO pre-processing tools by SPECIM. The radiance data were atmospherically corrected using the QUAC tools of the Harris ENVI software. The results were smoothed using a filter width of 4 and a degree of smoothing polynomial of 2 of the THOR Spectral Smoothing tools of ENVI. Regions of interest (ROIs) were extracted from the image to account for the spectral variation in the scene. The Spectral Angle Mapper implementation of ENVI software was used to classify the image using the ROIs. In the georeferencing process, the flight line image was placed on its actual position on the ground. Georectification and georeferencing of the AISA flight line images was performed by using the position and attitude data collected from the time synchronized on-board IMU/GPS navigation sensor. Images produced with CaliGeoPRO were ready for atmospheric correction, if required, and application requirements.

\section{RESULTS}

\subsection{Results of UAV-based 3D laser scanning}

The first tests of the LiDAR payload were conducted in April 2015 over two hectares of an airport near Lucerne. The recorded point cloud data represent a part of the runway with adjacent meadow and a hangar covered by grass. A part of the scene is shown in Fig. 5 in the processed orthoimagery. This scene is depicted as a 3D colorized point cloud in Fig. 6. The results demonstrate high quality of the final point cloud and orthoimagery and are comparable with other similar systems. The positional and vertical accuracy $(1 \sigma)$ of the post-processed flight line was $0.02 \mathrm{~m}$ and $0.025 \mathrm{~m}$, respectively. The orientation accuracy of roll and pitch was 0.05 degrees and 0.11 degrees of yaw.

\subsection{Results of UAV-based hyperspectral scanning}

The hyperspectral payload integration was tested in October 2015. The resulting hyperspectral imagery in Fig. 7 shows the area sensed during a calibration test flight as orthorectified image in natural colours. Figure 8 portrays part of the sensed area in false colours as a combination of two bands from visible and one from near infrared spectrum depicting the differences between vegetation cover. The accuracy of the flight line trajectory was similar as reported for the lidar mission and it is 
demonstrated by the seamless merge of three image data strips which accurately preserved the geometry of landscape features such as roads.

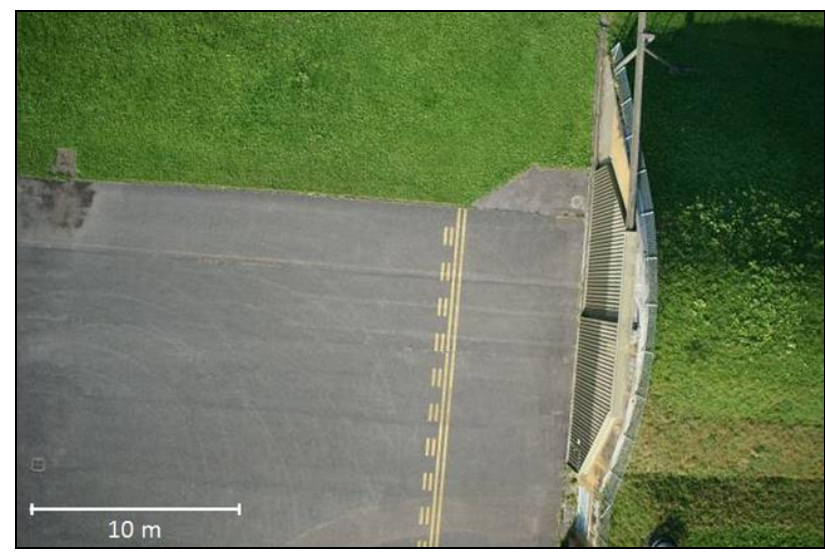

Figure 5: Orthoimagery of an airplane hangar (15 m wide) taken with the camera integrated in the laser scanning payload.

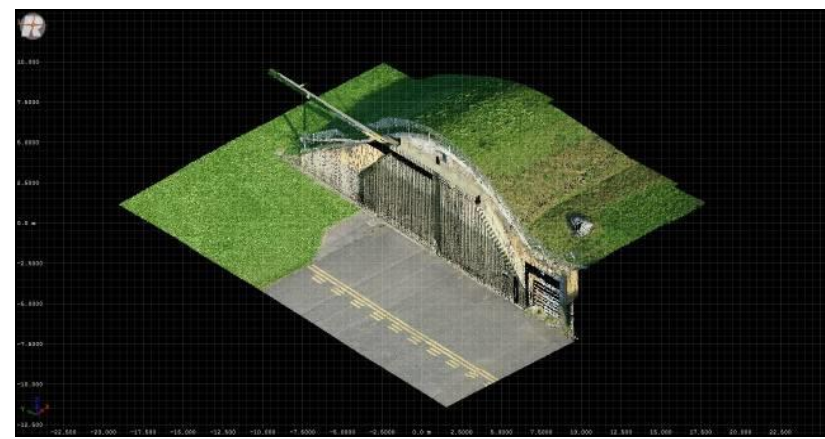

Figure 6: Section of the LiDAR point cloud representing the airplane hangar in Fig. 5 after post-processing.

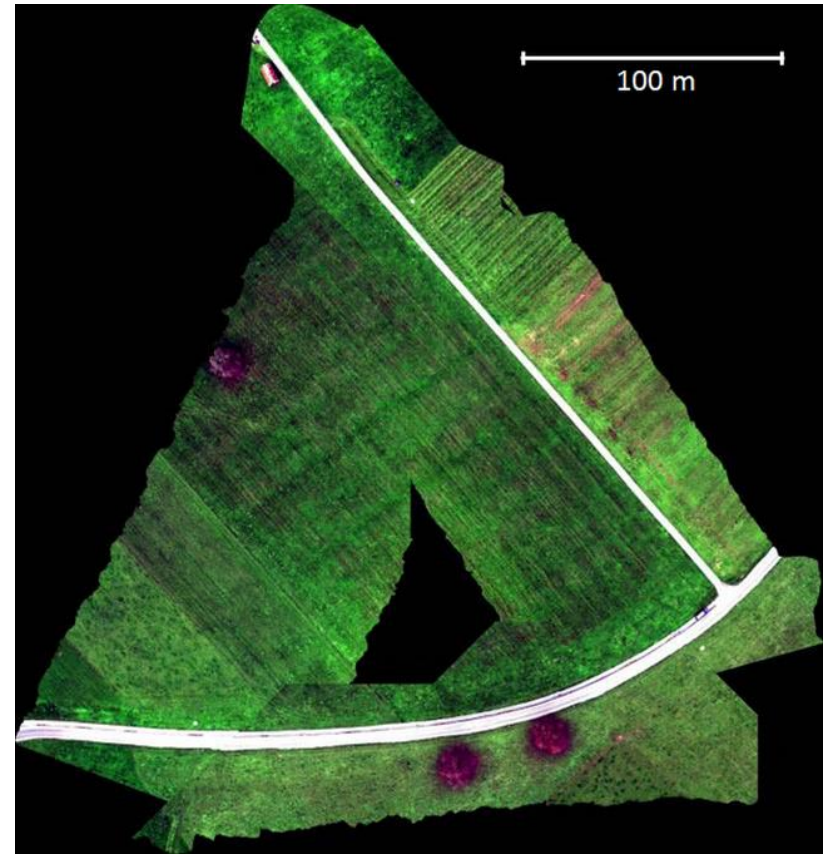

\section{CONCLUSION}

This paper introduced our custom integration of laser scanning and hyperspectral imaging payloads on a small UAV platform. It was demonstrated that the system is stable providing high resolution imagery and point clouds with a high geometric accuracy. The potential of coupling both technologies is very promising in applications where high resolution in spatial, spectral, and temporal domain is required and 3D geometric representation of the surface needed. Therefore, the main applicability of the presented UAS is in research of various aspects of landscape dynamics on local scale, such as monitoring landslides, soil or river bank erosion, vegetation growth, phenology, etc. Such applications of the UAS will be addressed in future research.

\section{ACKNOWLEDGEMENTS}

This paper originated with the financial support of the scientific project APVV-0176-12 funded by the Slovak Research and Development Agency and the projects VEGA 1/0473/14 and VEGA 1/0474/16 funded by the Slovak Research Grant Agency. We would like to thank Benedikt Imbach and Christoph Fallegger for assistance in preparing the flight missions. Furthermore, we want to thank SPECIM for the support of data processing and data visualization. 


\section{REFERENCES}

Aasen, H., Burkart, A., Bolten, A., Bareth, G., 2015. Generating 3D hyperspectral information with lightweight UAV snapshot cameras for vegetation monitoring: From camera calibration to quality assurance. ISPRS Journal of Photogrammetry and Remote Sensing, 108(10), pp. 245-259.

Bareth, G., Aasen, H., Bendig, J., Gnyp, M. L., Bolten, A., Jung, A., Michels, R. and Soukkamäki, J., 2015. Hyperspectral full-frame cameras for monitoring crops: spectral comparison with portable spectroradiometer measurements. Photogrammetrie - Fernerkundung - Geoinformation, Vol. 2015(1), pp. 69-79.

Black, M., Riley, T.R., Ferrier, G., Fleming, T.R. and Fretwell, P.T., 2016. Automated lithological mapping using airborne hyperspectral thermal infrared data: A case study from Anchorage Island, Antarctica. Remote Sensing of Environment, 176, pp. 225-241.

Colomina, I. and Molina, P., 2014. Unmanned aerial systems for photogrammetry and remote sensing: A review. ISPRS Journal of Photogrammetry and Remote Sensing, 92, pp. 79-97.

Eck, C. and Imbach, B., 2011. Aerial Magnetic Sensing with an UAV Helicopter. ISPRS - International Archives of the Photogrammetry, Remote Sensing and Spatial Information Sciences, Zürich, Switzerland, Vol. XXXVIII-1/C22(1).

Mandlburger, G., Wieser, M., Hollaus, M., Pfennigbauer, M. and Riegl, U., 2016. Multi-temporal UAV-borne LiDAR point clouds for vegetation analysis - a case study, Geophysical Research Abstracts, 18, EGU2016-7036-1. http://meetingorganizer.copernicus.org/EGU2016/EGU20167036-1.pdf (26 March 2016).

Ryan, J.P., Davis, C.O., Tufillaro, N.B., Kudela, R.M. and Gao, B.-C. 2014. Application of the Hyperspectral Imager for the Coastal Ocean to Phytoplankton Ecology Studies in Monterey Bay, CA, USA. Remote Sensing, 6, 1007-1025.

Sima, A., Baeck, P.-J., Delalieux, S., Livens, S., Blommaert, J., Delauré, B. and Boonen, M. (2016). A new COmpact hyperSpectral Imaging system (COSI) for UAS. Geophysical Research Abstracts, 18, EGU2016-5504, http://meetingorganizer.copernicus.org/EGU2016/EGU20165504.pdf (26 March 2016)

Shendryk, I., Tulbure, M., Broich, M., McGrath, A., Alexandrov, S. and Keith, D., 2016. Mapping tree health using airborne laser scans and hyperspectral imagery: a case study for a floodplain eucalypt forest. Geophysical Research Abstracts, 18, EGU2016-355, 2016, http://meetingorganizer.copernicus.org/EGU2016/EGU2016355.pdf (25 March 2016).

Yang, B. and Chen, C., 2015. Automatic registration of UAVborne sequent images and LiDAR data. ISPRS Journal of Photogrammetry and Remote Sensing, 101, 262-274.

Wallace, L., Watson, C. and Lucieer, A., 2014. Detecting pruning of individual stems using Airborne Laser Scanning data captured from an Unmanned Aerial Vehicle. International Journal of Applied Earth Observation and Geoinformation, 30, pp. 76-85.
Zarco-Tejada, P.J., Guillén-Climent, M.L., HernándezClemente, R., Catalina, A., González, M.R. and Martín, P., 2013. Estimating leaf carotenoid content in vineyards using high resolution hyperspectral imagery acquired from an unmanned aerial vehicle (UAV), Agricultural and Forest Meteorology, 171-172(4), pp. 281-294. 